\title{
Optimized enzymatic synthesis of levulinate ester in solvent-free system.
}

\begin{abstract}
Ethyl levulinate, produced through esterification of levulinic acid, is a ketoester with various applications. Synthesis of ethyl levulinate was carried out in solvent-free system using immobilized Candida antarctica lipase B (Novozym 435) as the biocatalyst for the reaction. Response surface methodology (RSM) with a four-factor-five-level central composite rotatable design (CCRD) was employed to study and optimize the reaction conditions in the synthesis of levulinate ester. The effect of four main reaction parameters including time, temperature, ethanol/levulinic acid molar ratio and amount of enzyme on the synthesis of ester were analyzed. A quadratic polynomial model was fitted to the data with an R2 of 0.8993. Model validation experiments show good correspondence between actual and predicted values. A high conversion yield $(96.2 \%)$ was obtained at the optimum conditions of $51.4{ }^{\circ} \mathrm{C}, 41.9 \mathrm{~min}, 292.3 \mathrm{mg}$ enzyme amount and 1.1:1 alcohol:acid molar ratio.
\end{abstract}

Keyword: Levulinic acid; Enzymatic synthesis; Esterification; Solvent-free system; Response surface methodology. 